\title{
surgical treatment of Loffler endocarditis in an 8-year-old girl
}

\author{
Zhangke Guo ${ }^{1}$, Fan $\mathrm{Fan}^{1}$, Pei $\mathrm{Li}^{1}$, Xiaofeng $\mathrm{Li}^{1}$, Ning $\mathrm{Ma}^{1}$, Libing $\mathrm{Fu}^{1}$, Song $\mathrm{Bai}^{1}$, Feng \\ Tong ${ }^{1}$, Jia Zheng ${ }^{1}$, and Qilin $\mathrm{Li}^{1}$ \\ ${ }^{1}$ Beijing Children's Hospital
}

August 20, 2020

\begin{abstract}
Introduction Idiopathic hyper eosinophilic syndrome (HES) is a rare disease characterized by sustained increase in eosinophilia. Lesions can affect various organs and systems. The global prevalence rate is unknown, and the prognosis is poor. At present, most of the literature reports are based on cases. Heart involvement may be present in $50 \%$ to $60 \%$ of patients, this is called Loffler endocarditis. Loffler endocarditis is a serious complication of hyper eosinophilia syndrome, which is characterized by a special type of fibrotic endocarditis. Loffler endocarditis is an inflammatory cardiac condition characterized by eosinophilic infiltration in the heart. Methods In this article we report an 8-year-old girl who was diagnosed as having Loffler endocarditis in thrombotic phase and treated by surgery successfully. Results Our patient had a good prognosis during the follow up of half year. She had no symptoms of heart failure and echocardiography findings were normal. Discussion The cardiac damage occurs in three stage processes: the necrotic, thrombotic, and fibrotic stages. This unusual but sometimes life-threatening disease is often detected in the late phase, resulting in no curative strategy available to reverse the disease process. The overall prognosis of patients with Loffler endocarditis is very poor. Current treatments include anticoagulation and anti-eosinophils therapy,surgery only used in selected cases. Surgical treatment of HES in adolescents is very rare. The present case illustrates that with well controlled peripheral eosinophilia, proper surgical treatment in selected patients can improve their prognosis in the near future but long-term follow up is necessary.
\end{abstract}

\section{Introduction}

Idiopathic hyper eosinophilic syndrome (HES) is a rare disease characterized by sustained increase in eosinophilia. Lesions can affect various organs and systems. The global prevalence rate is unknown, and the prognosis is poor. At present, most of the literature reports are based on cases. Heart involvement may be present in $50 \%$ to $60 \%$ of patients, this is called Loffler endocarditis.[1]. Loffler endocarditis is a serious complication of hyper eosinophilia syndrome, which is characterized by a special type of fibrotic endocarditis. Loffler endocarditis is an inflammatory cardiac condition characterized by eosinophilic infiltration in the heart. The cardiac damage occurs in three stage processes: the necrotic, thrombotic, and fibrotic stages. This unusual but sometimes life-threatening disease is often detected in the late phase, resulting in no curative strategy available to reverse the disease process. The overall prognosis of patients with Loffler endocarditis is very poor. Current treatments include anticoagulation and anti-eosinophils therapy,surgery only used in selected cases. Surgical treatment of HES in adolescents is very rare. In this article we report an 8-year-old girl who was diagnosed as having Loffler endocarditis in thrombotic phase and treated by surgery successfully.

\section{Case report}

An 8-year-old girl was admitted to our hospital in December 2019 complaining of rash for 11 days, abdominal pain for 8 days and fever, cough for 3 days. On physical examination, we found symmetrical distribution of dense reddish skin rash on limbs, ears and buttocks, no fading of pressure, slightly higher than skin, partially fused into pieces. Laboratory studies showed a white blood cell count of $14.83 \times 10^{9} / \mathrm{L}$ with $41.9 \%$ 
eosinophils and an absolute value of $6.21 \times 10^{9} / \mathrm{L}$ (normal values, $0.5 \%-5 \%$ or $0.05-3 \times 10^{9} / \mathrm{L}$ ). A bone marrow biopsy showed marked eosinophilia with $42.5 \%$ but no evidence of abnormal myeloid maturation. Liver and kidney functions, troponin-I level, and creatinine kinase level were normal. Rheumatoid factor erythrocyte sedimentation rate, serum immunoglobulins and antinuclear antibodies were all within normal limits. The stool, urine and parasite analysis were normal. The X-ray of chest suggested pneumonia, abdominal ultrasound examination and transthoracic echocardiography showed normal. After 13 days of anti-infective and anti-eosinophils therapy, the white blood cell count of $16.83 \times 10^{9} / \mathrm{L}$ with $13.9 \%$ eosinophils and an absolute value of $1.58 \times 10^{9} / \mathrm{L}$. the patient still had intermittent fever with higher temperature of up to 38.5 degree. Appeared of facial edema, anorexia, swelling of the liver. The X-ray of chest suggested aggravated pneumonia and pleural effusion. Transthoracic echocardiography revealed a large mass which nearly obliterated the right ventricular cavity and extended into the right ventricular outflow tract. The right atrium enlarged, moderate tricuspid regurgitation was noted, and moderate pericardial effusion was observed (Figure 1). So we did an emergency surgery for this patient. We operated via median sternotomy and under cardiopulmonary bypass. We found a large thrombus involving almost the entire right ventricle (Figure 2), the tricuspid was surrounded by the thrombus tissue seriously, then thrombectomy, tricuspid valvuloplasty were performed. The duration of extracorporeal circulation was 107 minutes and the aortic blockage duration was 82 minutes. The patient was extubated 10 hours after the operation in the Cardiac Care Unit(CCU) and no further complications. We used antibiotics to prevent infections, Using of furosemide and spironolactone to reduce cardiac load, used low-molecular-weight heparin calcium to prevent thrombus recurrence.

Histologic specimen showed thrombus, all of the pathological tissue were pink, degeneration, no structure, local rich in mucus with scattered and focal neutrophils, with a small number of small lymphocytes, eosinophils (HE staining, original magnification x10) (Figure 3).

\section{Discussion}

There are obvious individual differences in the clinical manifestations of HES, ranging from asymptomatic, multiple organ involvement, and even death. This pathology may involve many organs and systems, such as the skin, lungs, nervous system, gastrointestinal tract, kidneys, and heart. Therefore, diagnosis may be a challenge. The main morbidity and mortality of HES patients are mostly cardiovascular complications. [2] Heart involvement may be present in $50 \%$ to $60 \%$ of patients, this is called Loffler endocarditis.

Due to infiltration and degranulation of eosinophils, toxic proteins are released, causing tissue inflammation and late fibrosis resulting in secondary damage to the myocardium and endocardium. It is usually characterized by thickening of the endocardium and dilation of the atrium. Doppler echocardiography can show hyperechoic mass in the heart cavity, suggesting fibrosis or thrombosis, and is often located in the apical area of the left and right ventricles. [3],[4]

Echocardiography and imaging studies may be the main tools for assessing cardiac anatomy and function. However, Loffler endocarditis requires pathological diagnosis, so endocardial biopsy remains the gold standard. In some cases, myocardial biopsy may be a dangerous operation. Therefore, clinicians should personally evaluate each individual before performing such invasive procedures. In addition, when evaluating the possibility of Loffler endocarditis, it is important to exclude the main differential diagnosis of HES, including hyper eosinophils secondary to hypersensitivity and parasitic infections. In this case, preoperative laboratory studies showed peripheral hyper eosinophilia and echocardiography showed typical intraventricular space-occupying changes. The diagnosis of Loffler endocarditis is basically established, and postoperative pathological specimens have confirmed the previous diagnosis. The pathological features of Loffler's endocarditis include thickening of endocardial fibers, leading to a decrease in cardiac volume, thrombosis and restrictive cardiomyopathy. The clinical manifestations are heart failure, thromboembolic events and atrial fibrillation.

The main purpose of treatment is to reduce the level of eosinophils in peripheral blood and tissues, prevent terminal organ damage, and avoid the occurrence of adverse thrombotic events. Heart failure in Loffler's endocarditis is mainly due to limited diastolic function of the heart. Treatment includes intravenous di- 
uretics to reduce the preload of the heart. In addition, for symptomatic patients, the first-line drugs of choice are corticosteroids, followed by cytotoxic drugs such as hydroxyurea, and immunomodulators such as alpha-interferon. Glucocorticoid therapy is clinically proven to be effective for HES, which can improve the condition of myocardial injury and normalize peripheral hyper eosinophilia. [5],[6]

Secondary treatment should target cardiac complications such as heart failure and the presence of intracardiac thrombosis. Sometimes, cardiovascular complications require surgical treatment. Surgical experience is limited to cases where the heart valve function of patients due to HES is affected, but in more serious cases, acute surgical intervention may be necessary. In this case reported by our center, due to the large amount of thrombus tissue filling the right ventricle in a short time, the volume of the right ventricle was significantly reduced, the tricuspid valve was wrapped in the thrombus tissue, the opening of tricuspid valve was restricted, and there was a risk of pulmonary embolism caused by thrombosis. Therefore, the heart thrombus was removed under emergency cardiopulmonary bypass surgery.

Our patient had a good prognosis during the follow up of half year. She had no symptoms of heart failure and echocardiography findings were normal. This result might be due to the proper operation as well as the control of peripheral eosinophilia before and after the surgical procedure. Loffler endocarditis caused by hyper eosinophilia syndrome is unusual and fatal in children. The present case illustrates that with well controlled peripheral eosinophilia, proper surgical treatment in selected patients can improve their prognosis in the near future but long-term follow up is necessary.

Ethics approval This study has been approved by Medical Ethics Committee of Beijing Children's Hospital, Capital Medical University and the composition and procedure of Medical Ethics Committee is accordance with GCP principal and national relevant laws and regulations

Number:2020-Z-062

\section{References}

1. Yong-fang Guo, Zhi-hong Han, Teng-yong Jiang, Wei Fang, Ran Dong, Xue-si Wu Chinese medical journal 2009 Dec 05;122(23):2914-6

2. Shuzhen Wang, Aiqin Wang, Bin Guo, Shaowei Zhu, Zhaofu Chi, Xiuhe Zhao Journal of stroke and cerebrovascular diseases : the official journal of National Stroke Association 2014 Jul;23(6):1709-12 doi:10.1016/j.jstrokecerebrovasdis.2013.10.023

3. Carla Lofiego, Marinella Ferlito, Guido Rocchi, Elena Biagini, Enrica Perugini, Angelo Branzi, Claudio Rapezzi European journal of heart failure 2005 Oct;7(6):1023-6 doi:10.1016/j.ejheart.2005.06.004

4. Giovanni C Salanitri AJR. American journal of roentgenology 2005 May;184(5):1432-3 doi:10.2214/ajr.184.5.01841432

5. Juan Benezet-Mazuecos, Adolfo de la Fuente, Pedro Marcos-Alberca, Jeronimo Farre American journal of hematology 2007 Oct;82(10):861-2 doi:10.1002/ajh.20957

6. Rasha Mohamed Abayazeed, Mohamed Ayman Abdel-Hay, Sara Elfwal, Mahmoud Hssanein European heart journal. Case reports 2018 Jun;2(2):yty030 doi:10.1093/ehjcr/yty030 

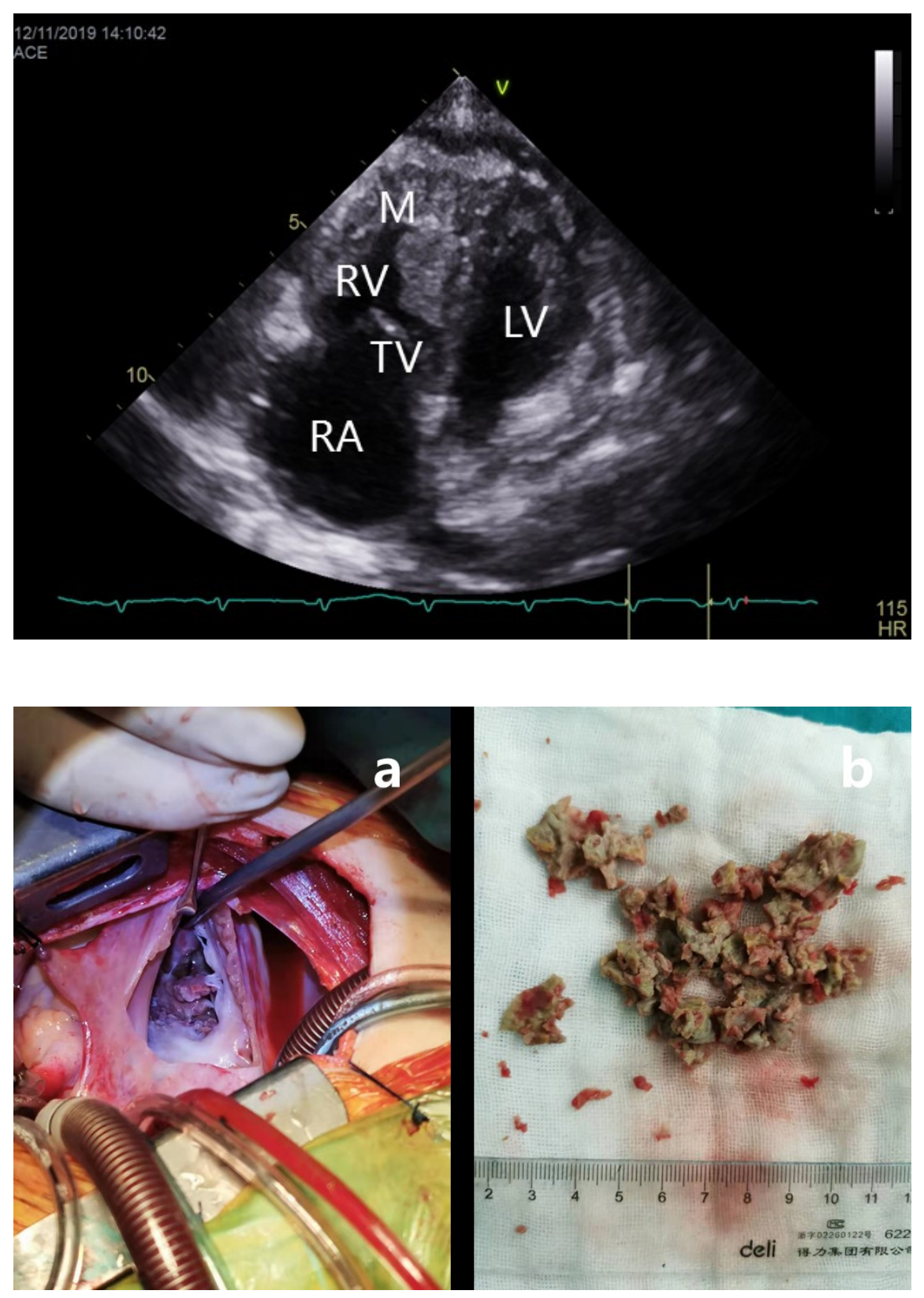


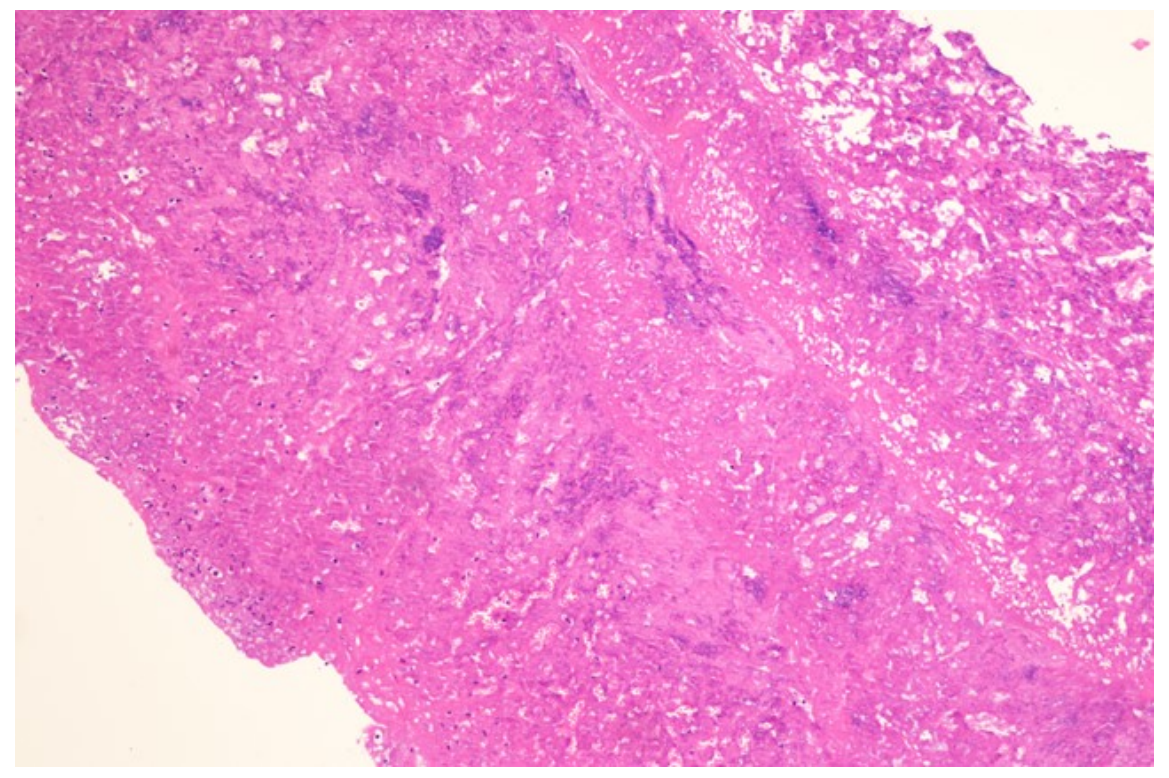

